\title{
Hooks at the Upper Instrumented Vertebra Can Adjust Postoperative Shoulder Balance in Patients with Adolescent Idiopathic Scoliosis: 5 Years or More of Follow-up
}

\author{
Shingo Kuroya ${ }^{1,2}$, Tsutomu Akazawa ${ }^{1,2}$, Toshiaki Kotani ${ }^{2}$, Tsuyoshi Sakuma ${ }^{2}$, Shohei Minami ${ }^{2}$, \\ Yoshiaki Torii ${ }^{1}$, Tasuku Umehara ${ }^{1}$, Masahiro Iinuma ${ }^{1}$, Kenichi Murakami ${ }^{1}$, Sumihisa Orita ${ }^{3}$, \\ Kazuhide Inage ${ }^{3}$, Yawara Eguchi ${ }^{3}$, Kazuki Fujimoto ${ }^{3}$, Yasuhiro Shiga ${ }^{3}$, Junichi Nakamura ${ }^{3}$, \\ Gen Inoue ${ }^{4}$, Masayuki Miyagi ${ }^{4}$, Wataru Saito ${ }^{4}$, Seiji Ohtori ${ }^{3}$, Hisateru Niki ${ }^{1}$

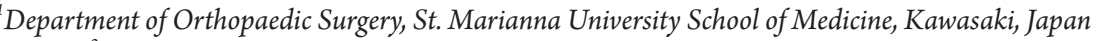 \\ ${ }^{2}$ Department of Orthopedic Surgery, Seirei Sakura Citizen Hospital, Sakura, Japan \\ ${ }^{3}$ Department of Orthopaedic Surgery, Graduate School of Medicine, Chiba University, Chiba, Japan \\ ${ }^{4}$ Department of Orthopaedic Surgery, Kitasato University School of Medicine, Sagamihara, Japan
}

Study Design: A retrospective cohort study.

Purpose: This study aims to investigate postoperative shoulder imbalance (PSI) $\geq 5$ years postoperatively in patients who underwent posterior spinal fusion using hooks at the upper instrumented vertebra (UIV) for Lenke type 1 adolescent idiopathic scoliosis (AIS).

Overview of Literature: Studies have reported PSI due to excessive correction of the main thoracic curve.

Methods: We examined 56 patients with AIS who underwent a posterior spinal fusion with hooks at the UIV from 2004 to 2010. Of these, we enrolled 14 patients who underwent surgery, at least, 5 years ago. X-rays and Scoliosis Research Society-22 (SRS-22) questionnaire were administered. To evaluate the shoulder balance, T1 vertebral tilt angle (T1 tilt), clavicle angle, and radiographic shoulder height (RSH) were measured. PSI was considered as the absolute value of the postoperative RSH being $\geq 20 \mathrm{~mm}$. Based on radiographs obtained immediately postoperatively, we divided patients into two groups as follows: the balanced group (absolute value of $\mathrm{RSH}<20 \mathrm{~mm}$ ) and imbalanced group (absolute value of $\mathrm{RSH} \geq 20 \mathrm{~mm}$ ).

Results: The frequency of PSI was $28.6 \%$ immediately postoperatively, $0 \% 2$ years postoperatively, and $7.1 \%$ at the last follow-up. In the balanced group, PSI did not occur even at 2 years postoperatively or at the last follow-up. In the imbalanced group, PSI was improved in all patients 2 years postoperatively and all patients, except one patient, at the last follow-up. No significant differences were noted in the frequency of distal adding-on at 2 years postoperatively or the last follow-up between the balanced group and the imbalanced group. We observed moderate negative correlations between the absolute value of T1 tilt and the SRS-22 pain and satisfaction at the last follow-up.

Conclusions: Hooks at the UIV could adjust the shoulder balance to avoid long-term PSI in patients with AIS.

Keywords: Adolescent idiopathic scoliosis; Long-term follow-up; Postoperative shoulder imbalance; Shoulder balance; Hook of the upper instrumented vertebra

Received Aug 10, 2018; Revised Dec 10, 2018; Accepted Jan 10, 2019

Corresponding author: Tsutomu Akazawa

Department of Orthopaedic Surgery, St. Marianna University School of Medicine, 2-16-1 Sugao, Miyamae-ku, Kawasaki, Kanagawa, 2168511, Japan

Tel: +81-44-977-8111, Fax: +81-44-977-9651, E-mail: cds00350@par.odn.ne.jp 


\section{Introduction}

Since spinal fusions for adolescent idiopathic scoliosis (AIS) are performed on adolescent patients, its appearance is one of the crucial concerns, along with back pain, function, and quality of life [1-3]. Although shoulder imbalance is a critical concern after spinal fusions, the prevalence of postoperative shoulder imbalance (PSI) is reportedly as high as $25 \%$ [4]. Reportedly, the self-image of the shoulder balance in postoperative patients correlates with $\mathrm{X}$-ray evaluation [5]. Of note, achieving shoulder balance is necessary to improve patient satisfaction.

Lately, segmental pedicle screw fixation has been extensively used, and a study has reported improvement in the correction rate [6]. In addition, PSI due to excessive correction of the main thoracic (MT) curve has been reported [4]. Namikawa et al. [7] highlighted the significance of avoiding the shoulder balance defects immediately postoperatively, as PSI does not change after 29-month followup. In addition, Matsumoto et al. [8] reported that hybrid methods using hooks and pedicle screws led to markedly lower T1 tilt angle and clavicle angle (CA) 2 years postoperatively, compared with segmental pedicle screw fixations; this study suggested that, compared with rigid pedicle screw fixation, flexible hooks are better for adjusting the shoulder balance in the long-term.

This study aims to investigate changes in the shoulder balance and health-related quality of life $\geq 5$ years postoperatively in patients who underwent posterior spinal fusion using hooks at the upper instrumented vertebra (UIV) for Lenke type 1 AIS. We hypothesize that hooks at the UIV could adjust the shoulder balance to avoid longterm PSI.

\section{Materials and Methods}

\section{Subjects}

This study protocol was approved by the Institutional Review Board of Seirei Sakura Citizen Hospital (IRB approval no., No. 29-0145). We examined 56 patients with AIS who underwent a posterior spinal fusion with hooks at the UIV from 2004 to 2010. The inclusion criteria were as follows: (1) Lenke type 1 curve, (2) aged 10-19 years at the time of surgery, and (3) $\geq 5$-year follow-up. The exclusion criteria were as follows: (1) diagnosis other than AIS, (2) use of pedicle screws at the UIV, (3) fixation including the proximal thoracic (PT) curve, (4) reoperative cases, and (5) history of spinal surgery before AIS surgery. We tried to contact all 56 patients by postal mail. Of these, we excluded the following patients: 21 patients who failed to respond to the mails, nine patients who refused to participate in this research, one patient who underwent anterior spinal fusion before posterior spinal fusion, seven patients with Lenke type 2 curve, and four patients with Lenke type 6 curve. Hence, we enrolled 14 patients (all females; average age at surgery, $15.0 \pm 1.9$ years [range, $12-19$ years]; average follow-up period, 7.6 years [range, 5.9-11.5 years]) who fulfilled the inclusion criteria and agreed to participate in this research. Written informed consents were obtained. All cases were right thoracic curves. Lenke types were as follows: $1 \mathrm{~A}(\mathrm{n}=11), 1 \mathrm{~B}(\mathrm{n}=2)$, and $1 \mathrm{C}(\mathrm{n}=1)$. The UIV were as follows: T3 $(\mathrm{n}=1)$, T4 $(\mathrm{n}=8), \mathrm{T} 5(\mathrm{n}=4)$, and T7 $(\mathrm{n}=1)$. The lower instrumented vertebra (LIV) were as follows: T11 $(\mathrm{n}=1), \mathrm{L} 1(\mathrm{n}=6), \mathrm{L} 2(\mathrm{n}=3), \mathrm{L} 3(\mathrm{n}=2)$, and L4 $(\mathrm{n}=2)$. The fusion levels were T3-L1 ( $\mathrm{n}=1), \mathrm{T} 4-\mathrm{T} 11(\mathrm{n}=1), \mathrm{T} 4-$ L1 ( $\mathrm{n}=5)$, T4-L2 ( $\mathrm{n}=1), \mathrm{T} 4-\mathrm{L} 4(\mathrm{n}=1)$, T5-L2 $(\mathrm{n}=2), \mathrm{T} 5-$ L3 $(n=2)$, and T7-L4 $(n=1)$. For all patients, X-rays of the whole spine were examined, and the Scoliosis Research Society-22 (SRS-22) questionnaire was distributed. X-ray images were taken standing upright from the front and the side, preoperatively, immediately postoperatively ( 1 week postoperatively or the first images obtained when patients were able to stand upright), 2 years postoperatively, and at the last follow-up ( $\geq 5$ years postoperatively). Furthermore, SRS-22 was evaluated at the last follow-up.

\section{Measurement variables}

In the frontal view, we measured Cobb angles of the PT curve, MT curve, and lumbar curve. In addition, the coronal balance (distance between a perpendicular line to the $\mathrm{C} 7$ and the midline of the sacrum) was measured from the frontal view (right, positive; left, negative). In the lateral view, we measured the thoracic kyphosis (TK) angle (T5-T12 angle), lumbar lordosis (LL) angle (L1S1 angle), and sagittal vertical axis (SVA; the distance between $\mathrm{C} 7$ and the posterior superior corner of S1). In addition, T1 vertebral tilt angle (T1 tilt), CA, and radiographic shoulder height (RSH) were measured from the frontal view to evaluate the shoulder balance. We defined the T1 tilt as the angulation of the upper endplate of T1 to the horizontal line. The CA was defined by the angulation of a horizontal line and the tangential line connecting the 
two highest points of each clavicle. The RSH was defined as the difference in the soft-tissue shadow directly superior to the acromioclavicular joint. A CA, T1 tilt, and RSH were positive $(+)$ when the left side was raised but negative (-) when the right side was raised [9]. In addition, PSI was considered as the absolute value of postoperative RSH being $\geq 20 \mathrm{~mm}$. The distal adding-on was considered as defined by Wang et al. [10] — if the deviation increased by $>5 \mathrm{~mm}$ from the central sacral vertical line of the vertebral body distal to instrumentation, or if the angle of the first intervertebral disc distal to instrumentation was increased by $>5^{\circ}$.

\section{Statistical analyses}

For statistical analyses, IBM SPSS Statistics ver. 22.0 (IBM Corp., Armonk, NY, USA) was used. Using a paired $t$-test, we compared preoperative and postoperative values. In addition, the Pearson correlation coefficient was used to determine correlations between shoulder balance parameters of X-ray images and SRS-22 subdomain scores. Based on radiographs obtained immediately postoperatively, we divided patients into two groups as follows: the balanced group (absolute value of RSH $<20 \mathrm{~mm}$ ) and imbalanced group (absolute value of RSH $\geq 20 \mathrm{~mm}$ ). Furthermore, we used the Mann-Whitney $U$-test or Fisher's exact test to compare the balanced group and imbalanced group. The level of significance in this study was set at $<5 \%$.

\section{Surgical procedure}

Surgery was performed in a prone position with midline skin incision. Typically, exposure was from the UIV through the LIV. In the left side UIV, claw hooks, including a transverse process hook and a facet hook, were placed. In the right side UIV, a transverse process hook was placed. Pedicle screws were placed in the remaining vertebral bodies. In cases of narrow pedicles, we skipped the level or installed ultra-high molecular weight polyethylene tapes using a sublaminar wiring method instead of pedicle screws. While placing anchors, scoliosis was corrected using a derotation maneuver with the left-sided rod. After the right-sided rod installation, we corrected concavity with distraction force and convexity with compression force.
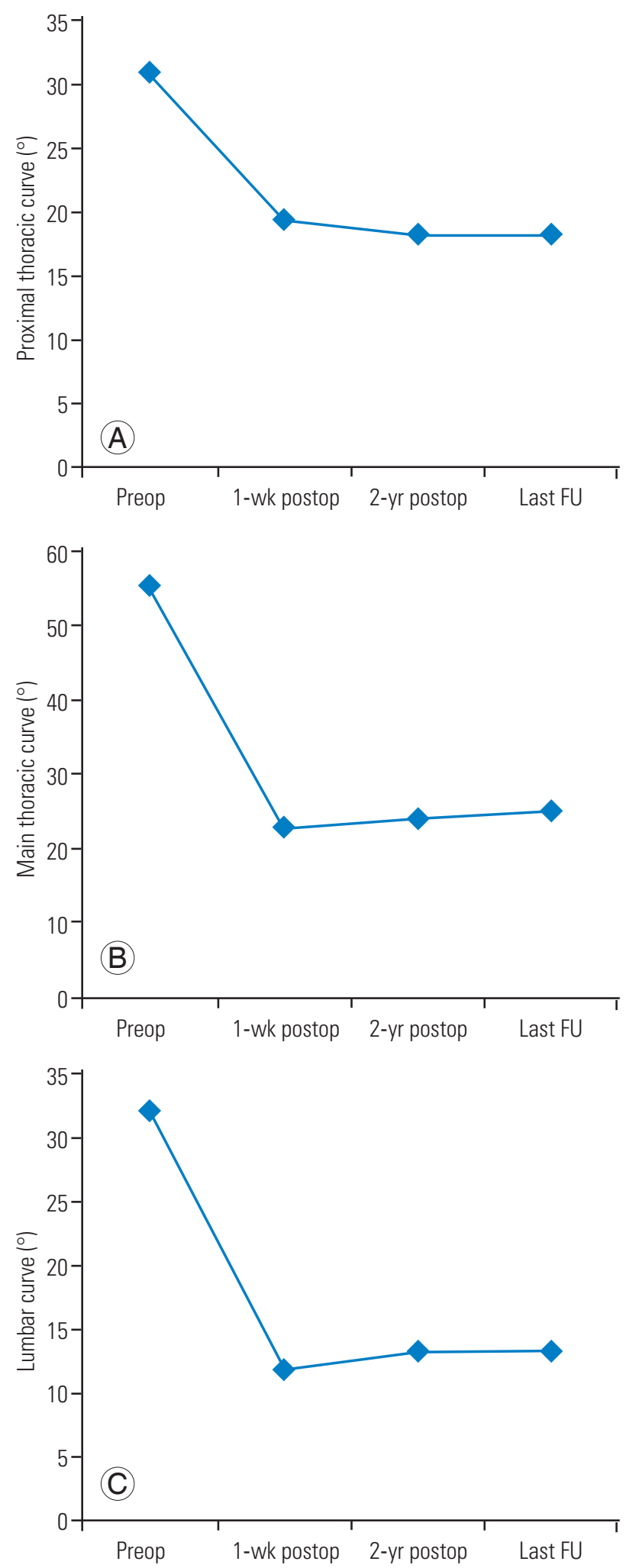

Fig. 1. Spinal coronal alignment parameters. Cobb angle of proximal thoracic curve (A), main thoracic curve (B), and lumbar curve (C). Preop, preoperative; Postop, postoperative; FU, follow-up. 


\section{Results}

\section{Spinal alignment parameters}

$\mathrm{X}$-ray analysis revealed that MT curves were $55.6^{\circ} \pm 9.5^{\circ}$ preoperatively, $22.8^{\circ} \pm 7.7^{\circ}$ immediately postoperatively, $23.9^{\circ} \pm 8.4^{\circ} 2$ years postoperatively, and $25.2^{\circ} \pm 7.4^{\circ}$ at the last follow-up (all postoperative values: $p<0.001$, compared with the preoperative value). We observed a significant loss of correction from 2 years postoperatively to the last follow-up of $2.3^{\circ}(p=0.031)$. PT curves were $30.9^{\circ} \pm 5.7^{\circ}$ preoperatively, $19.4^{\circ} \pm 8.2^{\circ}$ immediately postoperatively, $18.3^{\circ} \pm 6.5^{\circ} 2$ years postoperatively, and $18.3^{\circ} \pm 4.4^{\circ}$ at the last follow-up (all postoperative values: $p<0.001$, compared with the preoperative value). Lumbar curves were $32.1^{\circ} \pm 8.2^{\circ}$ preoperatively, $11.5^{\circ} \pm 4.4^{\circ}$ immediately postoperatively, $12.9^{\circ} \pm 4.5^{\circ} 2$ years postoperatively, and $12.9^{\circ} \pm 4.7^{\circ}$ at the last follow-up (all postoperative values: $p<0.001$, compared with preoperative value) (Fig. 1). The coronal balance was $-0.2 \pm 10.0 \mathrm{~mm}$ preoperatively, $-5.0 \pm 11.9 \mathrm{~mm}$ immediately postoperatively, $-7.1 \pm 11.2$ $\mathrm{mm} 2$ years postoperatively, and $4.0 \pm 23.6 \mathrm{~mm}$ at the last follow-up. No significant differences were noted at postoperative values compared with the preoperative value ( $p=0.114$, immediately postoperatively; $p=0.857,2$ years postoperatively; $p=0.718$, at the last follow-up).

TK was $10.5^{\circ} \pm 11.3^{\circ}$ preoperatively, $12.7^{\circ} \pm 4.6^{\circ}$ immediately postoperatively, $15.4^{\circ} \pm 8.5^{\circ} 2$ years postoperatively, and $17.4^{\circ} \pm 7.9^{\circ}$ at the last follow-up; this last follow-up value significantly increased compared with the preoperative value $(p=0.007)$. LL was $48.0^{\circ} \pm 13.5^{\circ}$ preoperatively, $38.2^{\circ} \pm 12.0^{\circ}$ immediately postoperatively, $51.2^{\circ} \pm 9.7^{\circ} 2$ years postoperatively, and $52.6^{\circ} \pm 8.4^{\circ}$ at the last follow-up. Although this measurement declined significantly from preoperatively to immediately postoperatively $(p=0.002)$, it significantly increased from immediately postoperatively to 2 years postoperatively $(p<0.001)$; no significant difference was noted at the last follow-up compared with the preoperative value $(p=0.076)$. The SVA was $-8.0 \pm 19.9 \mathrm{~mm}$ preoperatively, $17.6 \pm 37.9 \mathrm{~mm}$ immediately postoperatively, $-17.2 \pm 21.5 \mathrm{~mm} 2$ years postoperatively, and $-27.9 \pm 19.0$ $\mathrm{mm}$ at the last follow-up. Although this measurement increased immediately postoperatively ( $p=0.068)$, it significantly decreased at the last follow-up $(p=0.010)$ compared with the preoperative period.

\section{Shoulder balance parameters}

The RSH was negative (right high shoulder) in 12 patients preoperatively; of these, the absolute RSH values were $<10 \mathrm{~mm}$ in five patients, $10-20 \mathrm{~mm}$ in four patients, and $>20 \mathrm{~mm}$ in three patients. The RSH was positive (left high shoulder) in two patients preoperatively, who had the $\mathrm{RSH}<10 \mathrm{~mm}$. The PSI frequency was $28.6 \%$ immediately postoperatively, $0 \% 2$ years postoperatively, and $7.1 \%$ at the last follow-up. T1 tilt was $-2.9^{\circ} \pm 4.0^{\circ}$ preoperatively, $1.8^{\circ} \pm 3.2^{\circ}$ immediately postoperatively, $1.5^{\circ} \pm 4.2^{\circ} 2$ years postoperatively, and $0.5^{\circ} \pm 4.2^{\circ}$ at the last follow-up (all postoperative values: $p<0.001$, compared with the preoperative value). In addition, this parameter changed significantly and became horizontal 2 years postoperatively to last follow-up $(p=0.033)$. CA was $-2.3^{\circ} \pm 4.0^{\circ}$ preoperatively, $1.9^{\circ} \pm 2.6^{\circ}$ immediately postoperatively, $1.0^{\circ} \pm 1.7^{\circ}$ 2 years postoperatively, and $1.2^{\circ} \pm 2.0^{\circ}$ at the last followup (all postoperative values: $p<0.001$, compared with the preoperative value). All measurements were significantly different compared with the preoperative measurement (all $p<0.01$ ), but no significant long-term change was observed compared with measurements from immediately postoperatively. The RSH was $-11.2 \pm 9.4 \mathrm{~mm}$ preoperatively, $9.4 \pm 12.8 \mathrm{~mm}$ immediately postoperatively, $3.2 \pm 7.7$ $\mathrm{mm} 2$ years postoperatively, and $7.5 \pm 11.1 \mathrm{~mm}$ at the last follow-up (all postoperative values: $\mathrm{p}<0.001$, compared with the preoperative value) (Fig. 2). Nevertheless, we observed no significant long-term changes compared with measurements from immediately postoperatively.

\section{Comparisons between the balanced group and the imbalanced group}

In this study, 10 patients were present in the balanced group and four in the imbalanced group. Based on X-ray parameters of spinal alignment obtained preoperatively, we observed no significant differences in the PT curve $\left(29.9^{\circ} \pm 5.5^{\circ}\right.$ in the balanced group; $33.3^{\circ} \pm 6.2^{\circ}$ in the imbalanced group; $p=0.374)$, MT curve $\left(54.8^{\circ} \pm 7.9^{\circ}\right.$ in the balanced group; $57.5^{\circ} \pm 14.0^{\circ}$ in the imbalanced group; $p=0.839)$, lumbar curve $\left(32.6^{\circ} \pm 8.6^{\circ}\right.$ in the balanced group; $31.0^{\circ} \pm 8.2^{\circ}$ in the imbalanced group; $p=0.945$ ), TK $\left(9.0^{\circ} \pm 13.0^{\circ}\right.$ in the balanced group; $14.2^{\circ} \pm 4.6^{\circ}$ in the imbalanced group; $p=0.454)$, LL $\left(45.8^{\circ} \pm 15.5^{\circ}\right.$ in the balanced group; $53.0^{\circ} \pm 6.5^{\circ}$ in the imbalanced group; $p=0.330)$, or SVA $(-8.0 \pm 22.9 \mathrm{~mm}$ in the balanced group; 

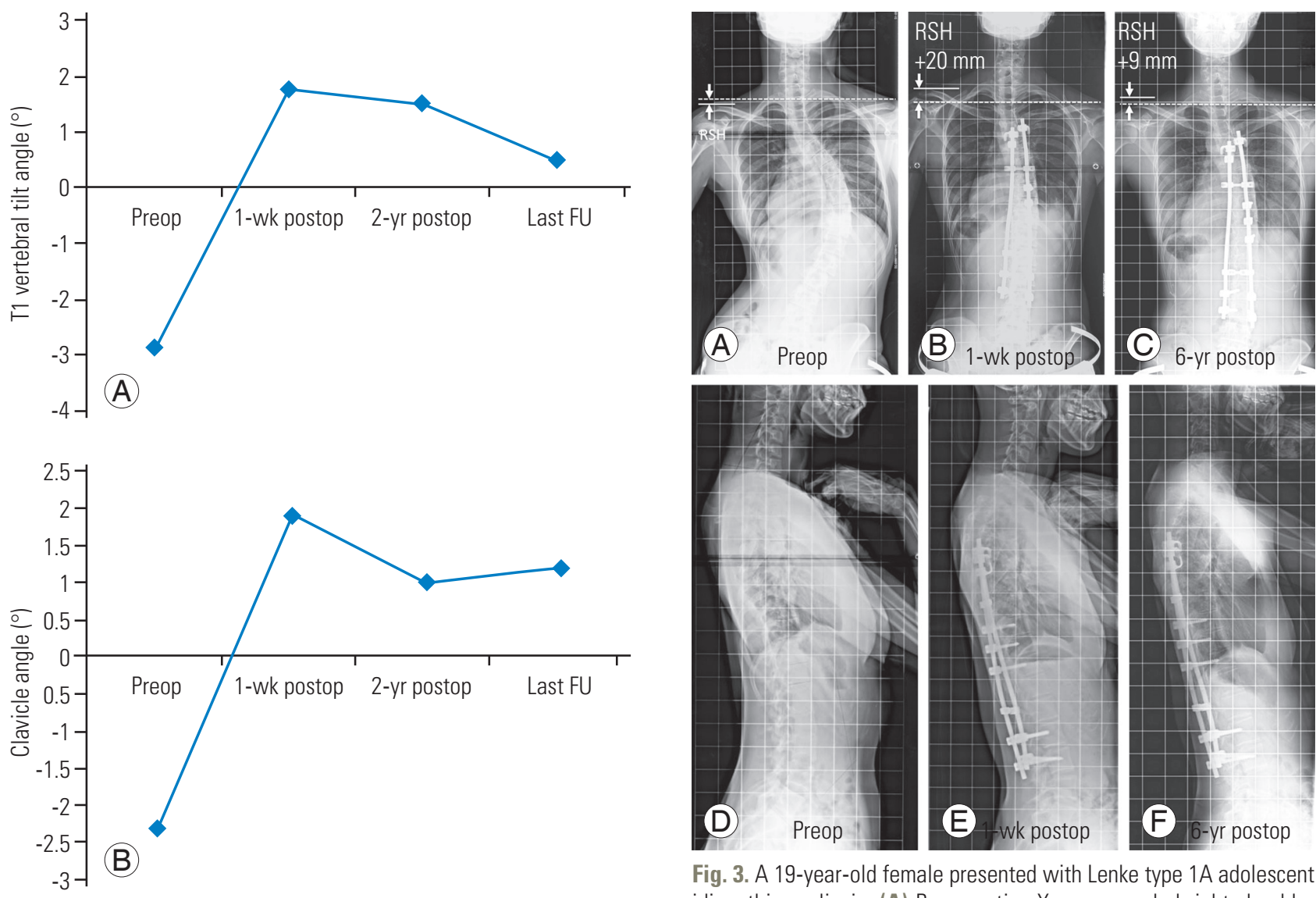

Fig. 3. A 19-year-old female presented with Lenke type 1A adolescent idiopathic scoliosis. (A) Preoperative $\mathrm{X}$-rays revealed right shoulder elevation with a $67^{\circ} \mathrm{MT}$ curve and $\mathrm{RSH}-6 \mathrm{~mm}$. (B) Although the MT curve had been corrected to $33^{\circ}$ immediately postoperatively (1 week postoperatively), RSH changed to $+20 \mathrm{~mm}$, with the left shoulder higher than the right. (C) At the latest follow-up (6 years postoperatively), the MT curve was $34^{\circ}$, and the RSH had improved to $+9 \mathrm{~mm}$. (D) The lateral view of preoperative $X$-ray showed an $11^{\circ} \mathrm{TK}$ and a $50^{\circ} \mathrm{LL}$. (E) The lateral view of postoperative 1 -week $\mathrm{X}$-ray showed a $16^{\circ} \mathrm{TK}$ and a $48^{\circ} \mathrm{LL}$. (F) The lateral view of postoperative 6 -year $\mathrm{X}$-ray showed a $14^{\circ} \mathrm{TK}$ and a $48^{\circ} \mathrm{LL}$. MT, main thoracic; $\mathrm{RSH}$, radiographic shoulder height; LL, lumbar lordosis; TK, thoracic kyphosis; Preop, preoperative; Postop, postoperative.

$-7.1 \pm 6.5 \mathrm{~mm}$ in the imbalanced group; $p=0.374)$. Based on X-rays obtained immediately postoperatively, no significant differences were noted in the correction rate of PT curves (37.6\% in the balanced group; $39.4 \%$ in the imbalanced group; $p=0.945)$, MT curves (59.3\% in the balanced group; $59.9 \%$ in the imbalanced group; $p=0.839$ ), or $-7.9 \pm 11.6 \mathrm{~mm}$ in the imbalanced group; $p=0.839$ ). Based on X-ray parameters of the shoulder balance obtained preoperatively, no significant differences were noted in T1 tilt $\left(-3.3^{\circ} \pm 4.4^{\circ}\right.$ in the balanced group; $-2.0^{\circ} \pm 2.8^{\circ}$ in the imbalanced group; $p=0.733)$, CA $\left(-2.7^{\circ} \pm 1.9^{\circ}\right.$ in the balanced group; $-3.3^{\circ} \pm 4.4^{\circ}$ in the imbalanced group; $p=0.142)$, or RSH $(-12.9 \pm 10.1 \mathrm{~mm}$ in the balanced group; lumbar curves $(60.0 \%$ in the balanced group; $70.6 \%$ in the imbalanced group; $p=0.240$ ).

In the balanced group, PSI did not occur even at 2 years postoperatively or the last follow-up. In the imbalanced group, PSI improved in all patients 2 years postoperatively and all patients, except one patient, at the last followup (Figs. 3, 4). We observed no significant differences 
between the balanced and imbalanced groups in the frequency of distal adding-on at 2 years postoperatively

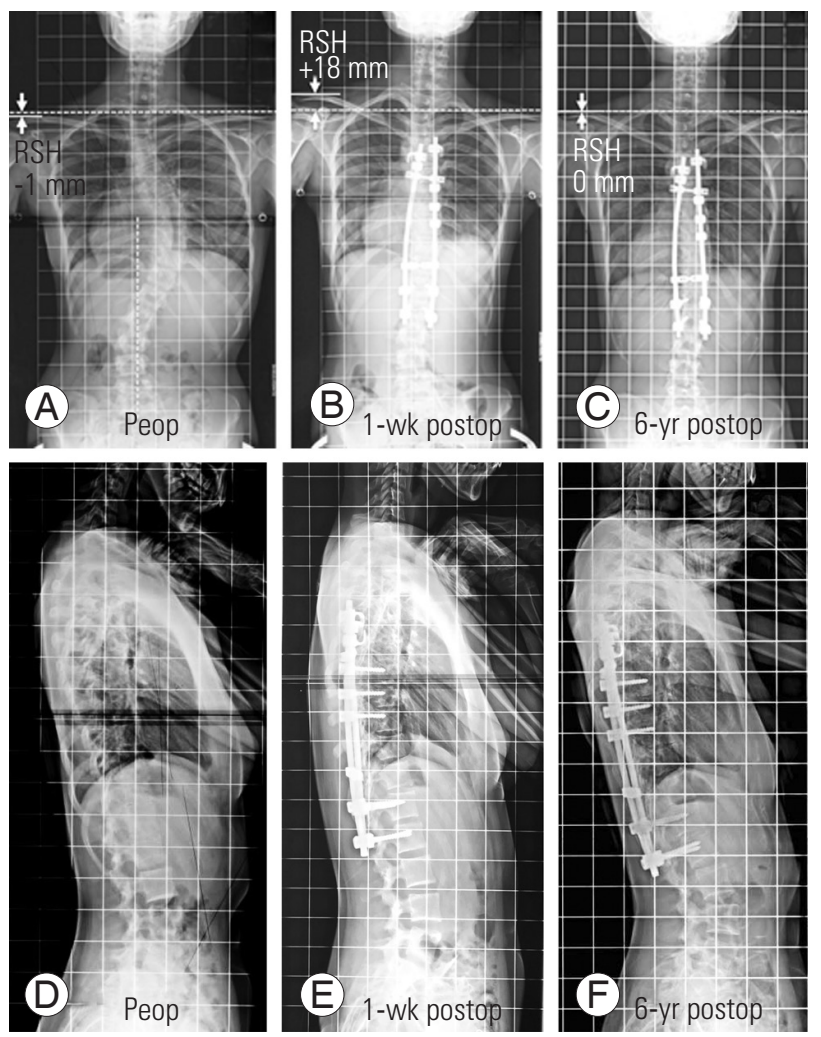

Fig. 4. A 16-year-old female presented with Lenke type 1A adolescent idiopathic scoliosis. (A) Preoperative $\mathrm{X}$-rays revealed right shoulder elevation with a $52^{\circ} \mathrm{MT}$ curve and $\mathrm{RSH}-1 \mathrm{~mm}$. (B) Although the MT curve had been corrected to $25^{\circ}$ immediately postoperatively (1 week postoperatively), RSH changed to $+18 \mathrm{~mm}$, with the left shoulder higher than the right. (C) At the latest follow-up (6 years postoperatively), the MT curve was $26^{\circ}$, and the RSH had improved to $+0 \mathrm{~mm}$. (D) The lateral view of preoperative $X$-ray showed a $9^{\circ} \mathrm{TK}$ and a $36^{\circ} \mathrm{LL}$. (E) The lateral view of postoperative 1 -week $X$-ray showed a $13^{\circ}$ TK and a $42^{\circ} \mathrm{LL}$. (F) The lateral view of postoperative 6 -year $\mathrm{X}$-ray showed a $17^{\circ} \mathrm{TK}$ and a $48^{\circ} \mathrm{LL}$. MT, main thoracic; $\mathrm{RSH}$, radiographic shoulder height; LL, lumbar lordosis; TK, thoracic kyphosis; Preop, preoperative; Postop, postoperative.
(30\% in the balanced group; $0 \%$ in the imbalanced group; $p=0.505)$ or the last follow-up ( $20 \%$ in the balanced group; $50 \%$ in the imbalanced group; $p=0.520$ ).

\section{Correlation between Scoliosis Research Society-22 and shoulder balance}

We assessed the correlation between SRS-22 and absolute value of T1 tilt, CA, and RSH at the last follow-up. Moderate negative correlations were observed between T1 tilt and pain $(r=-0.456, p=0.101)$ and satisfaction $(r=-0.491$, $p=0.075)$ scores, but no other significant correlations were noted. We observed no significant correlation between any subdomain scores of SRS-22 and CA or RSH (Table 1).

\section{Comparison between participants and non-partici- pants}

We compared the demographics of 14 participants enrolled in this study with 42 non-participants. No statistically significant differences were observed in the patients' age at surgery (participants, 15.0 \pm 2.0 years; non-participants, $15.4 \pm 1.9$ years; $p=0.467$ ), number of fused vertebral bodies (participants, 10.4 \pm 1.1 ; non-participants, $10.3 \pm 1.5$; $p=0.435)$. Comparison of the proportion of participants and non-participants based on sex revealed no significant difference between these groups in the percentage of females (participants, 100\%; non-participants, $95.2 \%$; $p=0.559)$.

\section{Discussion}

Factors that can affect shoulder balance after AIS reportedly include the degree of the lumbar spine curvature and

Table 1. Correlation between Scoliosis Research Society-22 and absolute value of T1 tilt, CA, and RSH at last follow-up

\begin{tabular}{|c|c|c|c|c|c|c|}
\hline & \multicolumn{2}{|c|}{ Absolute value of $\mathrm{T} 1$ tilt } & \multicolumn{2}{|c|}{ Absolute value of CA } & \multicolumn{2}{|c|}{ Absolute value of RSH } \\
\hline & $r$ & $p$-value & $r$ & $p$-value & $r$ & $p$-value \\
\hline Function & -0.302 & 0.294 & 0.261 & 0.368 & -0.176 & 0.548 \\
\hline Pain & -0.456 & 0.101 & 0.062 & 0.833 & -0.138 & 0.638 \\
\hline Self image & -0.326 & 0.256 & 0.217 & 0.456 & -0.251 & 0.386 \\
\hline Mental & -0.307 & 0.286 & -0.139 & 0.636 & -0.287 & 0.320 \\
\hline Satisfaction & -0.491 & 0.075 & 0.174 & 0.552 & -0.120 & 0.683 \\
\hline
\end{tabular}

Pearson correlation coefficient was used.

$\mathrm{CA}$, clavicle angle; $\mathrm{RSH}$, radiographic shoulder height. 
the PT and MT curves correction rates [4]. To prevent PSI, the PT curve must be adequately corrected while avoiding excessive corrections of the MT and lumbar curves. However, to date, no literature was available on how hooks at the UIV might affect PSI postoperatively. In this study, even when PSI occurred in the immediate postsurgical period, it resolved over the postoperative course, demonstrating that the use of hooks at the UIV could exert a positive impact in correcting the shoulder balance in the long-term.

Distal adding-on is a phenomenon observed in the lumbar vertebrae of AIS patients postoperatively and could result in inadequate clinical outcomes of the Lenke 1A curve or present a risk factor for repeat surgery [10]. While shoulder balance might improve postoperatively in PSI patients, distal adding-on reportedly occurs occasionally [8]. Distal adding-on has even been referred to as the compensation for PSI. In this study, patients who presented with PSI postoperatively experienced gradual improvement of shoulder balance during the postoperative course, but distal adding-on did not necessarily occur more frequently in these patients compared with those without PSI. We speculate the hooks at the UIV corrected shoulder balance in patients with PSI immediately postoperatively and that this was not the result of distal adding-on.

In the Lenke 1 curve, various factors have been reported as risk factors of PSI [4,5,7,8,11-13]. Changes in the PT, MT, and lumbar curves from preoperative to postoperative affected the postoperative shoulder balance in a metaanalysis [4]. Excessive correction of the MT curve is a momentous cause of PSI. As the scoliosis correction rate has amplified in recent years, surgeons must remember that the MT curve should not be overcorrected [14]. In addition, the postoperative behavior of the lumbar curve, including the distal adding-on, affects the postoperative shoulder balance. With shoulder imbalance immediately postoperatively, distal adding-on occurs and improves the shoulder balance [8]. Conversely, the distal adding-on is often accompanied by an unsatisfactory clinical outcome and a high risk of operation [10]. Furthermore, the preoperative angle and flexibility of the PT curve affect the shoulder balance [7].

During surgery, it is challenging to adjust the corrections of these three curves (PT, MT, and lumbar curves) while considering the shoulder balance. As the meticulous assessment of shoulder balance requires X-ray in the standing position, it is not as easy as described in the literature to adjust the shoulder balance in harmony with sufficient scoliosis corrections in the surgical field. Perhaps, hooks at the UIV shown in this study work with an autoregulator that adjusts the shoulder balance spontaneously postoperatively. Regarding PSI, it could solve the issue that surgeons waver whether or not to correct more during surgery.

This study has some limitations. The follow-up rate was very low (25\%). Only a few patients were available for observation up to $\geq 5$ years postoperatively, resulting in a low follow-up rate. In the future, we are considering additional attempts to follow-up with patients who did not reply or refused participation in this survey. As the control group treated with pedicle screws at the UIV was lacking, the effect of avoiding long-term PSI could not be revealed in this study. A study comparing the present results to those in patients treated with pedicle screws at the UIV is warranted. Furthermore, we plan to conduct another study involving long-term observation of patients treated with pedicle screws for comparison.

\section{Conclusions}

Preoperatively, the right shoulder had been higher in this study, but postoperatively, the left shoulder became higher. At 2 years postoperatively, the left shoulder remained higher, but after $\geq 5$ years, the MT curve had undergone loss of correction, and the T1 tilt became horizontal. The more level the T1 tilt, the higher the tendency for better patient satisfaction on the SRS-22 survey. Even when PSI was present in the immediate postoperative period, it improved by 2 years postoperatively and was maintained in $75 \%$ of patients after $\geq 5$ years postoperatively. Hooks at the UIV could adjust the shoulder balance to avoid longterm PSI in AIS patients. As the control patient group without hooks at the UIV was lacked, the effect of avoiding long-term PSI could not be distinctively revealed in this study. Hence, a study comparing the present results to those in patients treated with pedicle screws at the UIV is warranted.

\section{Conflict of Interest}

No potential conflict of interest relevant to this article was reported. 


\section{Author Contributions}

Shingo Kuroya and Tsutomu Akazawa wrote and prepared the manuscript, and all of the authors participated in the study design. All authors have read, reviewed, and approved the article.

\section{References}

1. Danielsson AJ, Nachemson AL. Back pain and function 23 years after fusion for adolescent idiopathic scoliosis: a case-control study-part II. Spine (Phila Pa 1976) 2003;28:E373-83.

2. Akazawa T, Minami S, Kotani T, Nemoto T, Koshi T, Takahashi K. Long-term clinical outcomes of surgery for adolescent idiopathic scoliosis 21 to 41 years later. Spine (Phila Pa 1976) 2012;37:402-5.

3. Akazawa T, Kotani T, Sakuma T, et al. Spinal fusion on adolescent idiopathic scoliosis patients with the level of L4 or lower can increase lumbar disc degeneration with sagittal imbalance 35 years after surgery. Spine Surg Relat Res 2017;1:72-7.

4. Zhang S, Zhang L, Feng X, Yang H. Incidence and risk factors for postoperative shoulder imbalance in scoliosis: a systematic review and meta-analysis. Eur Spine J 2018;27:358-69.

5. Kuklo TR, Lenke LG, Graham EJ, et al. Correlation of radiographic, clinical, and patient assessment of shoulder balance following fusion versus nonfusion of the proximal thoracic curve in adolescent idiopathic scoliosis. Spine (Phila Pa 1976) 2002;27:201320.

6. Suk SI, Lee SM, Chung ER, Kim JH, Kim SS. Selective thoracic fusion with segmental pedicle screw fixation in the treatment of thoracic idiopathic scoliosis: more than 5-year follow-up. Spine (Phila Pa 1976)
2005;30:1602-9.

7. Namikawa T, Matsumura A, Kato M, Hayashi K, Nakamura H. Radiological assessment of shoulder balance following posterior spinal fusion for thoracic adolescent idiopathic scoliosis. Scoliosis 2015;10:S18.

8. Matsumoto M, Watanabe K, Kawakami N, et al. Postoperative shoulder imbalance in Lenke type 1A adolescent idiopathic scoliosis and related factors. BMC Musculoskelet Disord 2014;15:366.

9. Blanke KM, Kuklo TR, Lenke LG, et al. Adolescent idiopathic scoliosis. In: O’Brien MF, editor. Radiographic measurementmManual. Memphis (TN): Medtronic Sofamor Danek Inc.; 2004. p. 55-7.

10. Wang Y, Hansen ES, Hoy K, Wu C, Bunger CE. Distal adding-on phenomenon in Lenke $1 \mathrm{~A}$ scoliosis: risk factor identification and treatment strategy comparison. Spine (Phila Pa 1976) 2011;36:1113-22.

11. Hong JY, Suh SW, Modi HN, Yang JH, Park SY. Analysis of factors that affect shoulder balance after correction surgery in scoliosis: a global analysis of all the curvature types. Eur Spine J 2013;22:1273-85.

12. Han X, Liu Z, Qiu Y, et al. Clavicle chest cage angle difference: is it a radiographic and clinical predictor of postoperative shoulder imbalance in Lenke I adolescent idiopathic scoliosis? Spine (Phila Pa 1976) 2016;41:1346-54.

13. Gotfryd AO, Silber Caffaro MF, Meves R, Avanzi O. Predictors for postoperative shoulder balance in Lenke 1 adolescent idiopathic scoliosis: a prospective cohort study. Spine Deform 2017;5:66-71.

14. Atici Y, Erdogan S, Akman YE, Mert M, Carkci E, Tuzuner T. The surgical overcorrection of Lenke type 1 deformities with selective fusion segments: what happens to the coronal balance? Korean J Spine 2016;13:151-6. 